\title{
Computer Program for obtaining theoretical X-ray Satellite Spectrum
}

\author{
Falguni Shrivastava ${ }^{1}$, Renuka Kendurkar ${ }^{1}$, B.D. Shrivastava ${ }^{1}$ and ${\operatorname{Ramji~} \operatorname{Yadav}^{2}}^{2}$ \\ ${ }^{1}$ School of Studies in Physics, Vikram University, Ujjain 456010, India \\ ${ }^{2}$ Institute of Computer Science, Vikram University, Ujjain 456010, India
}

Corresponding Author: rashmibasant@gmail.com Mob: 9893448358

Available online at: www.isroset.org

Received: 14/May/2018, Revised: 29/May/2018, Accepted: 09/Jun/2018, Online: 30/Jun/2018

\begin{abstract}
A computer program has been developed in TURBO $\mathrm{C}^{++}$to obtain theoretical X-ray satellite spectrum. Using the available theories for the origin of the X-ray satellite spectral lines, we calculate the energies and intensities of the different possible electronic transitions which give rise to the satellites. The energies and intensities of the transitions are fed in the developed computer program. The energy is taken as $\mathrm{X}$ and the intensity as $\mathrm{Y}$. The position of each line is taken to be the energy of transition on $\mathrm{X}$-axis and the height of each line is taken to be equal to the relative intensity on $\mathrm{Y}$-axis. Each spectral line corresponding to a transition is taken as a Gaussian line. The data for each Gaussian line is computed by the program. The data for composite spectrum of all the electronic transitions, i.e., for the envelope of all the Gaussian curves is computed by the program. The data obtained as the output of the program is plotted as a graph, using software Origin. The graph obtained is the theoretical X-ray satellite spectrum. This theoretical spectrum is compared with the available experimental results and the origin of the satellites is explained. In the present paper, we are reporting the algorithm, the developed computer program and the outputs obtained using the program. The program is versatile and can be used to obtain any theoretical spectrum when the energies and intensities of the transitions giving rise to the spectrum are known.
\end{abstract}

Key Words - Computer program in C++, X-ray satellites, spectra, Guassian curves, multiply ionized atom

\section{INTRODUCTION}

In an X-ray tube, $\mathrm{X}$-rays are generated by electron impact on a target and the radiation consists of both the continuous $\mathrm{X}$ rays and characteristic $\mathrm{X}$-rays. The spectrum of the radiation consists of characteristic spectrum of the target superimposed over the continuous spectrum. The spectral lines of the characteristic spectra resulting from transitions between atomic states, involving single vacancy are called diagram lines, because energy of such lines can be expressed as the difference of two terms in the single vacancy energy level diagram. The

diagram lines are generally found to be accompanied by groups of lines of slightly different energies and usually smaller intensities. The energies of these lines do not correspond to the energy difference between any two states of the normal single vacancy energy level diagram of the element concerned. These lines are known as X-ray satellite lines or non-diagram lines. [1-4]
Since the discovery of the X-ray satellites, the problem of finding the origin of these satellites has attracted attention of many workers and various theories have been put forward from time to time. It is well established that while the parent diagram line is emitted by a transition in the singly ionized atom, the X-ray satellite line is emitted due to transition in the multiply ionized atom. The X-ray satellite energy is the difference between the energies of initial and final states which are both doubly or multiply ionized. Thus, the satellite has a different energy than the energy of the Xray diagram line. [1-4]

We have undertaken research to find the origin of some particular unexplained X-ray satellites, i.e., to assign transitions in doubly ionized atom which give rise to such $\mathrm{X}$ ray satellites. [1-4] In our research, we have to calculate the theoretical X-ray satellite spectrum and then to compare it with the experimental spectrum. To obtain theoretical spectrum we calculate the energies of the different electronic transitions which are allowed between the initial and final double vacancy states and also the relative intensities of such transitions. After calculating the energies and intensities of all 
the possible electronic transitions, a composite spectrum is computed by taking energy on $\mathrm{X}$-axis and intensity on $\mathrm{Y}$ axis. The height of each line is taken to be equal to the relative intensity and the position of each line is taken to be the energy of transition. Each spectral line corresponding to a transition is taken as a Gaussian line. The width of all the spectral lines in the elements under consideration can be assumed to be equal and its value is decided by trial and error method. Depending upon the separation of the energies of the electronic transitions and the width of the Gaussian curve of each transition, the different Gaussian curves may overlap each other or may remain separate. The composite spectrum of all the electronic transitions, i.e., of all the Gaussian curves is obtained by computing an envelope of all the Gaussian curves. The envelope gives us the required theoretical spectrum. The computation has to be done in such a manner that the number of peaks obtained in the calculated spectrum is at least equal to or greater than the number of satellites observed experimentally in the spectrum of the element. This theoretical spectrum is compared with the available experimental results. The intense peaks are identified as the observed satellite lines. The transition assignments to the satellites based on the identification of the peaks, which arise due to the particular transitions, is then done. In this way, the origin of the satellites can be known.

The theoretical spectrum has been calculated with the help of a computer program. In the present paper, we are reporting the algorithm, the program and the outputs obtained using this program taking an example.

\section{THEORETICAL CONSIDERATIONS}

There are two steps through which the theoretical X-ray satellite spectrum can be obtained.

(1) For each of the transition in the multiply ionized atom a spectral line has to be drawn. This spectral line should have Gaussian curve shape. The intensity of this Gaussian curve should be the same as that of the transition. The center of the Gaussian curve should be at the energy of the transition. Such curves should be drawn for all the transitions considered for the whole satellite spectrum in a particular region of the $\mathrm{X}$ ray emission spectra.

(2) After obtaining all the individual spectrum for all the possible transition, an envelope has to be obtained of all the spectrum curves. This would be the required theoretical spectrum
The equation for Gaussian curve [5] is:

$$
\mathrm{Y}=\frac{1}{\sigma \sqrt{2 \pi}} \times \exp \left(-\frac{\mathbf{1}}{\mathbf{2}} \times\left(\frac{\mathrm{X}-\mu}{\sigma}\right)^{2}\right)
$$

where $\mu=$ mean, $\sigma=$ standered deviation, $\mathrm{X}$ and $\mathrm{Y}$ are the coordinates. In the present case $\mathrm{X}$ is the energy, $\mathrm{Y}$ is the relative intensity, $\mu$ is the energy of the particular transition about which a Gaussian curve will be centered. $\sigma$ determines the width of Gaussian curve, i.e., width of spectrum line. Actually, for a particular value of $\sigma$, the width of spectrum line at half of the maximum intensity is theoretically equal to $2.355 \sigma$.

In the present case, the maximum intensity of the Gaussian curve must be the intensity of the transition. We name this intensity as $\mathrm{I}_{\max }$ and modify eqn. (1) as follows:

$$
\mathrm{Y}=\mathrm{I}_{\max } \times \exp \left(-\frac{\mathbf{1}}{\mathbf{2}} \times\left(\frac{\mathrm{X}-\mu}{\sigma}\right)^{2}\right)
$$

In this equation, $\mu$ is the energy the particular transition and $I_{\max }$ is its intensity. $\sigma$ is chosen such that the width of each spectral line is such that the number of peaks obtained from the calculated spectrum is at least equal to or greater than the number of satellites observed experimentally in the spectrum of the element.

\section{DATA HANDLING FOR PROGRAM DEVELOPMENT}

(1) Input data file has two columns. First column is of the energy ( $\mu$, i.e., $\mathrm{mu})$ of a particular transition and second column is of the relative intensity $\left(\mathrm{I}_{\max }\right.$, i.e., Imax).

(2) There are a number of rows depending on the number of transitions considered for a particular satellite. We call them (No-of-Tran)

(3) In file Name variable is declared to handle input data file.

(4) The range of spectrum has to be decided by the user. On $\mathrm{X}$ axis, energy in $\mathrm{eV}$ has to be plotted. We call this value as $\mathrm{E}$. Hence, $\left(\mathrm{E}_{\min }\right.$, i.e., $\left.\mathrm{E}_{-} \min \right)$ and $\left(\mathrm{E}_{\max }\right.$, i.e., $\left.\mathrm{E}_{-} \max \right)$ are the values of $\mathrm{X}$ which decide the range.

(5) E has to be increased in step of (delta_E), which has to be decided by the user. Number of calculations of $\mathrm{Y}$ will be equal to $\left(\mathrm{E}_{\max }-\mathrm{E}_{\min }\right) /$ delta_E for each transition.

(6) Such calculation will be done for the number of transitions, i.e., No_of-Tran

(7) For any spectrum, the value of $\sigma$ (i.e., sigma) has to be decided by the user. The value of sigma decides the broadness of the Gaussian curve of each transition. 
(8) The output is obtained in the file which is given by the user and handled in output file Name variable in the program.

\section{ALGORITHM}

Step1: Declare and initialize various variables

Step2: Provide input ; Enter value of $\mathrm{E}_{\min }, \mathrm{E}_{\max }$, delta_E, sigma and in file Name, out file Name

Step3: Open files for reading /writing data

Step4: Initialize Y(relative intensity values as zeroes). Here Y is an array variable.

Step5: Calculate (by repetition) various $\mathrm{Y}$ values using equation (2).(See the implementation of equation (2) in program)

Step 6: Print array Y as output in out file Name.

\section{PROCEDURE FOR COMPUTING THEORETICAL X-RAY SATELLITE SPECTRAL DATA}

The performance of the computer program can be checked by taking an example. In the present paper, we are taking the example of an X-ray emission satellite spectrum in the L $\alpha$ region of the spectrum of element terbium $\left({ }_{65} \mathrm{~Tb}\right)$.[6] In table 1 are given the value of energies of 30 electronic transitions which are considered to be responsible for the considered Xray emission satellite. In this table, there are 30 rows corresponding to the 30 transitions. For each transition, the value of energy $\mu$ (in $\mathrm{eV}$ ) and relative intensity $\mathrm{I}_{\max }$ are given. This table is entered in a note-pad and the file is called Tb.txt. By pressing 'ctrl F9' the program is run.

The program asks for input as follows:

Enter E_min, E_max, delta_E, Sigma, No_ of.Tran

For the present example, we have entered the values as :

624063100.1130

After we have entered the value of No. of. Tran as 30 we get the command

Enter Input Filename

We write - Tb.txt

Then we get the command

Enter Output-File Name

We write - Tbout1 (This file is named as 'Tbout1'. Instead of 1 , it can be any other number according to our convenience)

Then we get the command

Tb.txt Tbout1 file exist

Illustration-This means that the range of the computed spectrum will be from 6240 to $6310 \mathrm{eV}$. The values of Y will be computed by increasing the value of energy 6240 by 0.1 $\mathrm{eV}$ and so on in each computation. The total number of data points for $\mathrm{Y}$ will be $(6310-6240) / 0.1=700$. As the value of $\sigma$ has been taken as 1 , the width of Gaussian curve for each transition at half maximum will be $2.355 \mathrm{eV}$. Computation will be complete for all the 30 transitions after 30 iterations. For each value of $\mathrm{Y}$, the 30 values for the 30 transitions will be added. The output will have only two columns. First column will have 700 data points corresponding to energy, starting from $6240 \mathrm{eV}$, ending with $6310 \mathrm{eV}$, each data point increasing by $0.1 \mathrm{eV}$. The second column will again have 700 data points, giving the value of $\mathrm{Y}$ for each of the energy data points.

By pressing enter key we come back to program

We go to 'File' and then to 'Quit'

We open the file in Note-pad. There are 700 data points in this file.

Out of the 700 data points of the output files, a portion of the output data is given in table 2 .

Table1. The energies of 30 electronic transitions, possible in the doubly ionized atom, and their relative intensities.[6]

\begin{tabular}{|c|c|c|}
\hline S.No. & Energy $(\mathrm{eV}) \quad(\mathrm{mu})$ & Relative Intensity(Imax) \\
\hline 1 & 6300.71 & 100 \\
\hline 2 & 6298.60 & 77.77 \\
\hline 3 & 6245.21 & 93.33 \\
\hline 4 & 6296.00 & 62.22 \\
\hline 5 & 6291.49 & 35 \\
\hline 6 & 6298.45 & 50 \\
\hline 7 & 6299.96 & 77.77 \\
\hline 8 & 6288.06 & 50 \\
\hline 9 & 6287.86 & 1.6 \\
\hline 10 & 6262.43 & 41.66 \\
\hline 11 & 6288.06 & 5.55 \\
\hline 12 & 6279.13 & 75 \\
\hline 13 & 6303.19 & 16.66 \\
\hline 14 & 6294.51 & 100 \\
\hline 15 & 6304.89 & $75^{*}$ \\
\hline 16 & 6288.41 & $25^{*}$ \\
\hline 17 & 6276.02 & 15.55 \\
\hline 18 & 6273.51 & 33.33 \\
\hline 19 & 6265.19 & 23.33 \\
\hline 20 & 6265.63 & 26.66 \\
\hline 21 & 6299.50 & 62.22 \\
\hline 22 & 6298.53 & 1.11 \\
\hline 23 & 6298.71 & 35 \\
\hline 24 & 6287.84 & 1.6 \\
\hline 25 & 6295.42 & 16.66 \\
\hline 26 & 6248.20 & $16.66 *$ \\
\hline 27 & 6288.01 & $11.11 *$ \\
\hline 28 & 6262.43 & 8.33 \\
\hline 29 & 6253.53 & 23.33 \\
\hline 30 & 6298.07 & $6.66^{*}$ \\
\hline
\end{tabular}

*Falguni's values 
Table 2. A portion of the output data file containing 100 data points out of the 700 data points. First column gives the value of $X$, (i.e energy) with increment of delta_E $=0.1$ and sigma $=1.0$. The second column gives the value of Y(i.e., relative density).

\begin{tabular}{|c|c|c|c|c|c|}
\hline S.No. & $\begin{array}{c}\mathrm{X} \\
\text { (Energy } \\
\text { in eV) }\end{array}$ & $\begin{array}{c}\mathrm{Y} \\
\text { Relative } \\
\text { intensity }\end{array}$ & S.No. & $\begin{array}{c}\mathrm{X} \\
\text { (Energy } \\
\text { in } \mathrm{eV})\end{array}$ & $\begin{array}{c}\mathrm{Y} \\
\text { Relative } \\
\text { intensity }\end{array}$ \\
\hline 518 & 6292.15 & 0 & 568 & 6297.16 & 0 \\
\hline 519 & 6292.25 & 0 & 569 & 6297.26 & 0 \\
\hline 520 & 6292.35 & 0 & 570 & 6297.36 & 0 \\
\hline 521 & 6292.45 & 0 & 571 & 6297.46 & 0 \\
\hline 522 & 6292.55 & 0 & 572 & 6297.56 & $1.2 \mathrm{E}-5$ \\
\hline 523 & 6292.65 & 0 & 573 & 6297.66 & 0.00129 \\
\hline 524 & 6292.75 & 0 & 574 & 6297.76 & 0.04893 \\
\hline 525 & 6292.85 & 0 & 575 & 6297.86 & 0.68358 \\
\hline 526 & 6292.95 & 0 & 576 & 6297.96 & 3.5063 \\
\hline 527 & 6293.05 & 0 & 577 & 6298.06 & 6.62408 \\
\hline 528 & 6293.15 & 0 & 578 & 6298.16 & 5.24472 \\
\hline 529 & 6293.25 & 0 & 579 & 6298.26 & 9.1126 \\
\hline 530 & 6293.35 & 0 & 580 & 6298.36 & 36.83726 \\
\hline 531 & 6293.45 & 0 & 581 & 6298.46 & 80.11576 \\
\hline 532 & 6293.55 & 0 & 582 & 6298.56 & 111.09434 \\
\hline 533 & 6293.65 & 0 & 583 & 6298.66 & 102.87436 \\
\hline 534 & 6293.75 & 0 & 584 & 6298.76 & 54.41255 \\
\hline 535 & 6293.85 & 0 & 585 & 6298.86 & 14.65548 \\
\hline 536 & 6293.95 & $1.8 \mathrm{E}-5$ & 586 & 6298.96 & 1.7652 \\
\hline 537 & 6294.05 & 0.00291 & 587 & 6299.06 & 0.0888 \\
\hline 538 & 6294.15 & 0.1712 & 588 & 6299.16 & 0.17933 \\
\hline 539 & 6294.25 & 3.69483 & 589 & 6299.26 & 3.31329 \\
\hline 540 & 6294.35 & 29.27769 & 590 & 6299.36 & 22.67358 \\
\hline 541 & 6294.45 & 85.17962 & 591 & 6299.46 & 56.96938 \\
\hline 542 & 6294.55 & 90.98953 & 592 & 6299.56 & 52.57943 \\
\hline 543 & 6294.65 & 35.68651 & 593 & 6299.66 & 18.62063 \\
\hline 544 & 6294.75 & 5.13894 & 594 & 6299.76 & 12.3937 \\
\hline 545 & 6294.85 & 0.27171 & 595 & 6299.86 & 46.534 \\
\hline 546 & 6294.95 & 0.00559 & 596 & 6299.96 & 77.76334 \\
\hline 547 & 6295.05 & 0.02043 & 597 & 6300.06 & 47.81476 \\
\hline 548 & 6295.15 & 0.48297 & 598 & 6300.16 & 10.79484 \\
\hline 549 & 6295.25 & 4.19944 & 599 & 6300.26 & 0.8986 \\
\hline 550 & 6295.35 & 13.40666 & 600 & 6300.36 & 0.2379 \\
\hline 551 & 6295.45 & 15.71475 & 601 & 6300.46 & 4.28782 \\
\hline 552 & 6295.55 & 6.76617 & 602 & 6300.56 & 32.03881 \\
\hline 553 & 6295.65 & 1.22673 & 603 & 6300.66 & 87.90295 \\
\hline 554 & 6295.75 & 3.11027 & 604 & 6300.76 & 88.54977 \\
\hline 555 & 6295.85 & 21.58744 & 605 & 6300.86 & 32.75128 \\
\hline 556 & 6295.95 & 56.12451 & 606 & 6300.96 & 4.4476 \\
\hline 557 & 6296.05 & 53.5781 & 607 & 6301.06 & 0.22176 \\
\hline 558 & 6296.15 & 18.77928 & 608 & 6301.16 & 0.00406 \\
\hline 559 & 6296.25 & 2.41673 & 609 & 6301.26 & $2.7 \mathrm{E}-5$ \\
\hline 560 & 6296.35 & 0.11419 & 610 & 6301.36 & 0 \\
\hline 561 & 6296.46 & 0.00198 & 611 & 6301.46 & 0 \\
\hline 562 & 6296.56 & $1.3 \mathrm{E}-5$ & 612 & 6301.56 & 0 \\
\hline 563 & 6296.66 & 0 & 613 & 6301.66 & 0 \\
\hline 564 & 6296.76 & 0 & 614 & 6301.76 & 0 \\
\hline 565 & 6296.86 & 0 & 615 & 6301.86 & 0 \\
\hline
\end{tabular}

\begin{tabular}{|l|l|l|l|l|l|}
\hline 566 & 6296.96 & 0 & 616 & 6302.06 & 0 \\
\hline 567 & 6297.06 & 0 & 617 & 6302.16 & 0 \\
\hline
\end{tabular}

In table 2, there are 100 data points and has two columns. First column gives the value of X, (i.e energy) with increment of delta $\mathrm{E}=0.1$ and sigma $=1.0$. The second column gives the value of Y(i.e., relative density).

We select the whole data and copy it in software Origin where we plot graph between X (i.e., energy) and Y (i.e., relative intensity).

\section{RESULTS AND DISCUSSION}

As already discussed in the introduction, the aim of developing this computer program is to obtain theoretical Xray satellite spectrum. The spectrum is the result of large number electronic transitions in a multiply ionized atom. Before we discuss the results obtained for a number of transitions using the developed program, it will be appropriate to first discuss the type of the Gaussian curve obtained for a single transition using the developed program. For plotting such a curve, we choose one of the 30 electronic transitions, namely, $\mathrm{L}_{3} \mathrm{M}_{3}{ }^{3} \mathrm{D}_{3} \rightarrow \mathrm{M}_{3} \mathrm{M}_{5}{ }^{3} \mathrm{~F}_{4}$, having maximum relative intensity $I_{\max }=100$ and energy position $\mu=6300.71 \mathrm{eV}$. [6] The program has been run from $E_{\min }=6290 \mathrm{eV}$ to $\mathrm{E}_{\max }=6310 \mathrm{eV}$, choosing $\Delta \mathrm{E}=0.1 \mathrm{eV}$. We get 200 data points in the output file using the program. We plot graph between $\mathrm{X}$ (i.e., Energy) and Y (i.e., Relative Intensity) in software Origin. Four curves have been drawn for $\sigma=0.5,1.0,1.5,2.0$ respectively. Fig. 1 gives the four Gaussian curves. These curves illustrate the effect of variation of $\sigma$. The curve for $\sigma=0.5$ is narrowest and that of $\sigma=0.1$ is broadest. The width of the curve at half maximum has been found to be $2.355 \times 2=4.7 \mathrm{eV}$ for $\sigma=2.0$, same as that required by the theory. Thus, by choosing proper value of $\sigma$, the width of the spectral line can be changed. The value of $\sigma$ actually depends on the width of energy levels.

Now let us discuss the results obtained for a spectra consisting of a number of X-ray satellites. The element for which spectra have been drawn is terbium $\left({ }_{65} \mathrm{~Tb}\right)$. The region of the spectra is from $6240 \mathrm{eV}$ to $6310 \mathrm{eV}$. There are 30 electronic transitions possible in the doubly ionized atom. The data for these transitions, i.e., the energies of these transitions and their relative intensities, given in table 1 , is written on the note-pad and the file is given the name Tb.txt. The computer program was run using this data as mentioned 
above. The output was obtained in the file named Tbout1. This output file has two columns, one for energy and another for relative intensity. The output file is fed in the software Origin for plotting the graph.

When we plot graph between X (i.e., energy) and Y (i.e., relative intensity) in software Origin, we get the graph for the X-ray satellite spectra which are reproduced in fig. 2. This graph, i.e., the L-emission satellite spectra is the envelope of 30 Gaussian curves for the 30 electronic transitions given in table 1. Fig. 2 has three graphs (a), (b) and (c). In these three graphs, $\Delta \mathrm{E}$ has been kept as $0.1 \mathrm{eV}$ and $\sigma$ has been varied, i.e., $1,0.1$ and 0 . 5. The output file has 700 data points in these three graphs. When $\sigma=1$, then we get 9 satellite lines, when $\sigma=0.5$, then we get 15 satellite lines and when $\sigma=0.1$, then we get 18 satellite lines. Similarly, we have drawn another set of graph given in fig.3. This figure again has three graphs (a), (b) and (c). In these three graphs, $\Delta \mathrm{E}$ has been kept as $0.03 \mathrm{eV}$ and $\sigma$ has been varied, i.e., 1, 0.1 and 0.5. The output file has 2333 data points in these three graphs. In fig. 3 also, when $\sigma=1$, then we get 9 satellite lines, when $\sigma=0.5$, then we get 15 satellite lines and when $\sigma=0.1$, then we get 18 satellite lines. Thus, we get the same number of satellite lines if $\Delta \mathrm{E}$ is changed from $0.1 \mathrm{eV}$ to $0.03 \mathrm{eV}$, i.e., when the data points are change from 700 to 2333. These graphs demonstrate that in the computer program developed in this work, we can vary the number of electronic transitions, the energy range of satellite spectra, the number of data points in this energy range and the width of each Gaussian line. All these variations are possible and demonstrate the versatility in the developed program. This helps in obtaining the desired number of theoretical satellites which may be equated to the number of satellites obtained experimentally.

\section{CONCLUSION}

The aim of developing this computer program is to obtain theoretical X-ray satellite spectrum. The computer program has been developed in TURBO

$\mathrm{C}^{++}$. In the present paper, we are reporting the algorithm, the developed computer program and the outputs obtained using the program. The X-ray satellite spectrum is the result of large number electronic transitions in a multiply ionized atom. Using the available theoretical methods, we calculate the energies and intensities of the different possible electronic transitions which give rise to the satellites. The energies and intensities of the transitions are fed in the developed computer program. The position of each line is taken to be the energy of transition as $\mathrm{X}$ and the height of each line is taken to be equal to the relative intensity as Y. Each spectral line, i.e., a graph between $\mathrm{X}$ and $\mathrm{Y}$, corresponding to a transition is taken as a Gaussian line. The data for each Gaussian line is computed by the program. The data for composite spectrum of all the electronic transitions, i.e., for the envelope of all the Gaussian curves is computed by the program. The data obtained as the output of the program is plotted as a graph, using software Origin. The graph obtained is the theoretical X-ray satellite spectrum. This theoretical spectrum is compared with the available experimental results and the origin of the satellites is explained.

The performance of the computer program has been checked by taking an example. In the present paper, we are taking the example of an X-ray emission satellite spectrum in the $\mathrm{L} \alpha$ region of the element terbium $\left({ }_{65} \mathrm{~Tb}\right)$. The Gaussian curve obtained for a single transition using the developed program illustrate the effect of variation of $\sigma$. Thus, by choosing proper value of $\sigma$, the width of the spectral line can be changed.

The theoretical spectra obtained for the considered example demonstrate the capabilities of the developed computer program. We can vary the number of electronic transitions, the energy range of satellite spectra, the number of data points in this energy range and the width of each Gaussian line. All these variations are possible and demonstrate the versatility of the developed program.

Though this program has been developed to obtain theoretical X-ray satellite spectrum involving a number of electronic transitions for which energies and intensities have been calculated using some theory, yet this program is very versatile and can be used to obtain any theoretical spectrum when the energies and intensities of the transitions giving rise to the spectrum are known. Also modifications can be incorporated in the program according to need. 


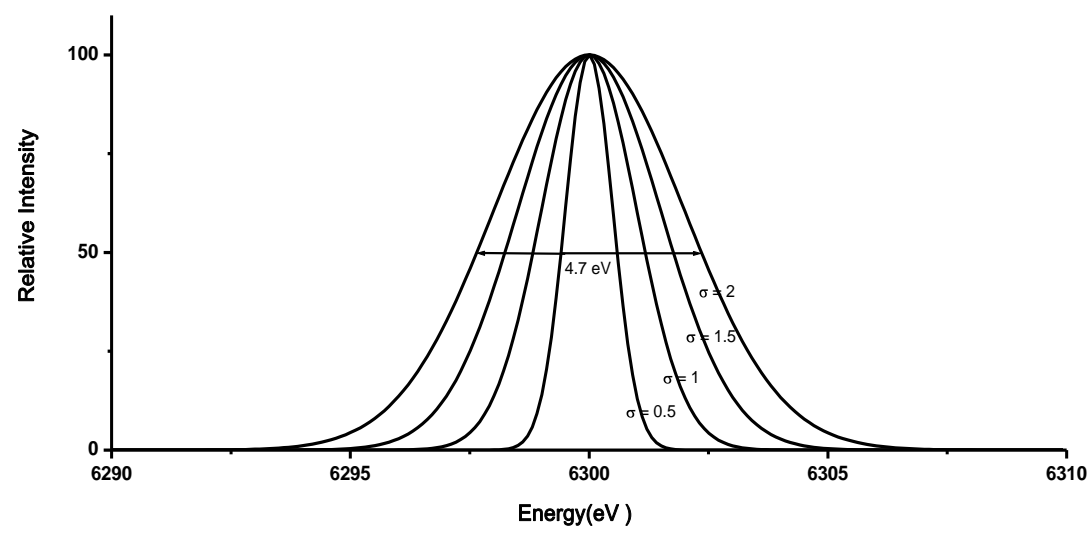

Fig.1. Gaussian curves for the electronic transition $\mathrm{L}_{3} \mathrm{M}_{3}{ }^{3} \mathrm{D}_{3} \rightarrow \mathrm{M}_{3} \mathrm{M}_{5}{ }^{3} \mathrm{~F}_{4}$ having maximum relative intensity $I_{\max }=100$ and energy position $\mu=6300.71 \mathrm{eV}$. The four curves have been drawn for $\sigma=0.5,1.0,1.5,2.0$ respectively, keeping $\Delta \mathrm{E}=0.1 \mathrm{eV}$ for all the curves. The width of the curve at half maximum is $2.355 \times 2=4.7 \mathrm{eV}$ for $\sigma=2.0$ as required by the theory.

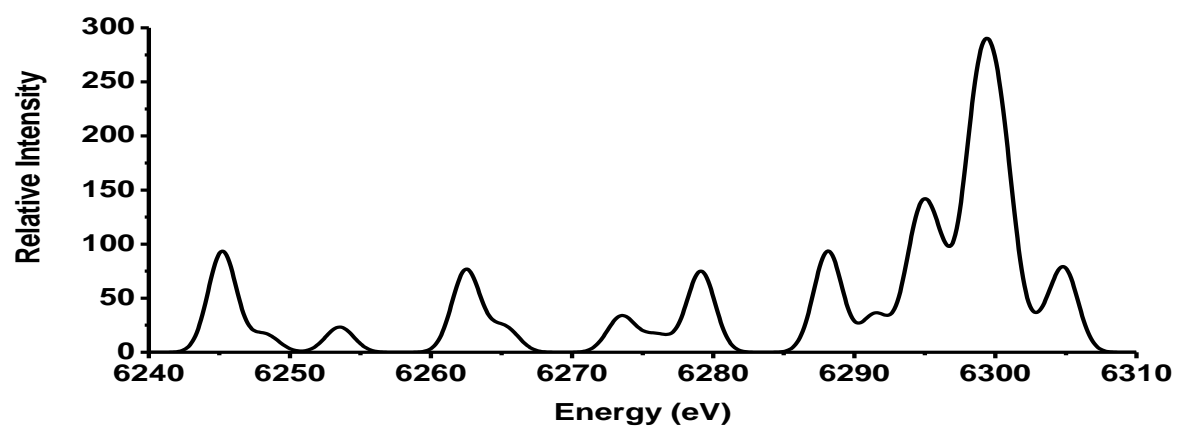

Fig. 2 (a) The L-emission satellite spectra obtained as the envelope of 30 Gaussian curves for the 30 electronic transitions given in table 1. The Gaussian curve for each of the transitions has $\sigma=1.0$ and $\Delta \mathrm{E}=0.1 \mathrm{eV}$.

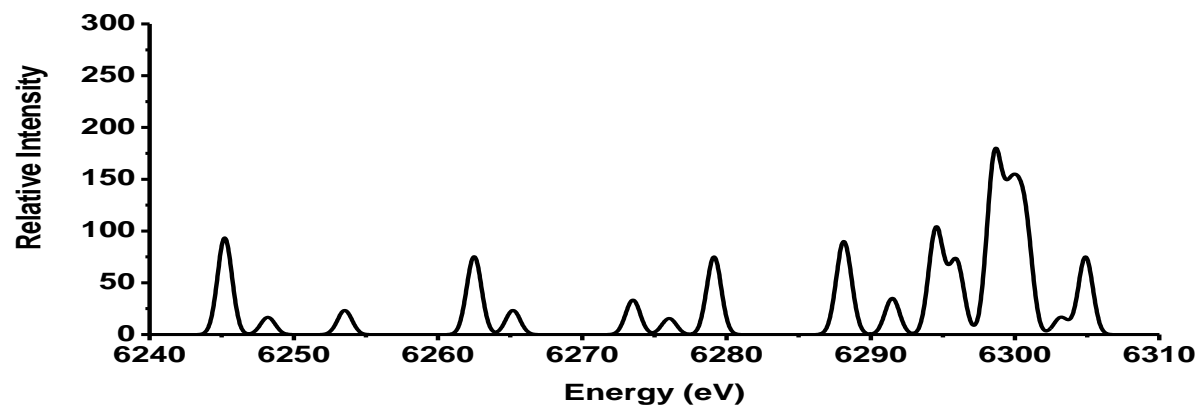

Fig.2 (b) The L-emission satellite spectra obtained as the envelope of 30 Gaussian curves for the 30 electronic transitions given in table 1 . The Gaussian curve for each of the transitions has $\sigma=0.5$ and $\Delta \mathrm{E}=0.1 \mathrm{eV}$. 


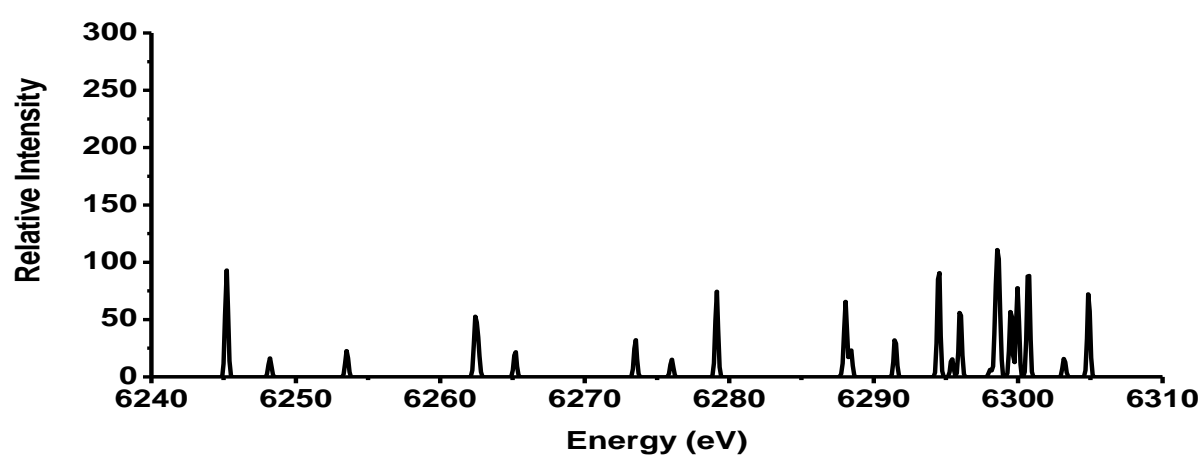

Fig.2(c) The L-emission satellite spectra obtained as the envelope of 30 Gaussian curves for the 30 electronic transitions given in table 1 . The Gaussian curve for each of the transitions has $\sigma=0.1$ and $\Delta \mathrm{E}=0.1 \mathrm{eV}$.

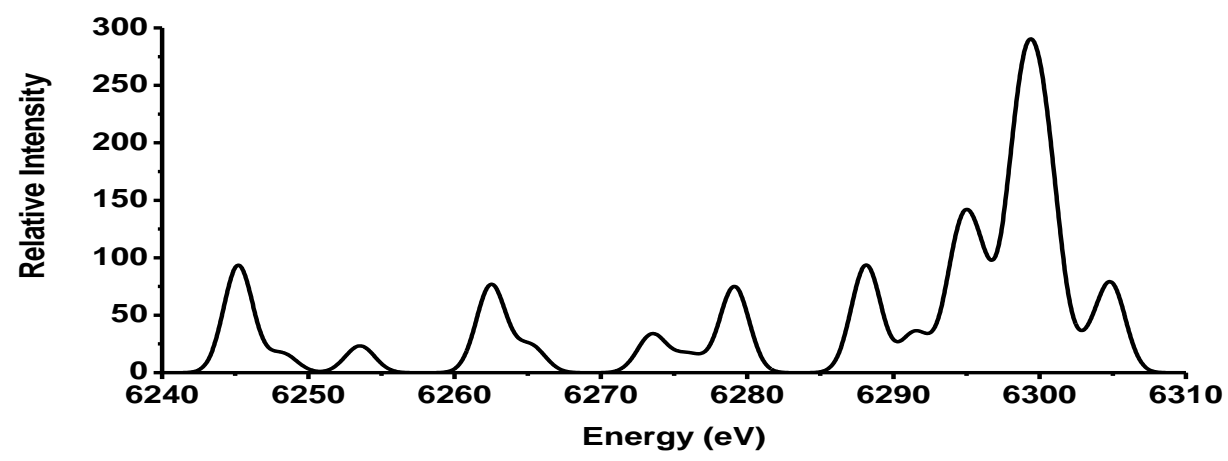

Fig.3 (a) The L-emission satellite spectra obtained as the envelope of 30 Gaussian curves for the 30 electronic transitions given in table 1. The Gaussian curve for each of the transitions has $\sigma=1.0$ and $\Delta \mathrm{E}=0.03 \mathrm{eV}$.

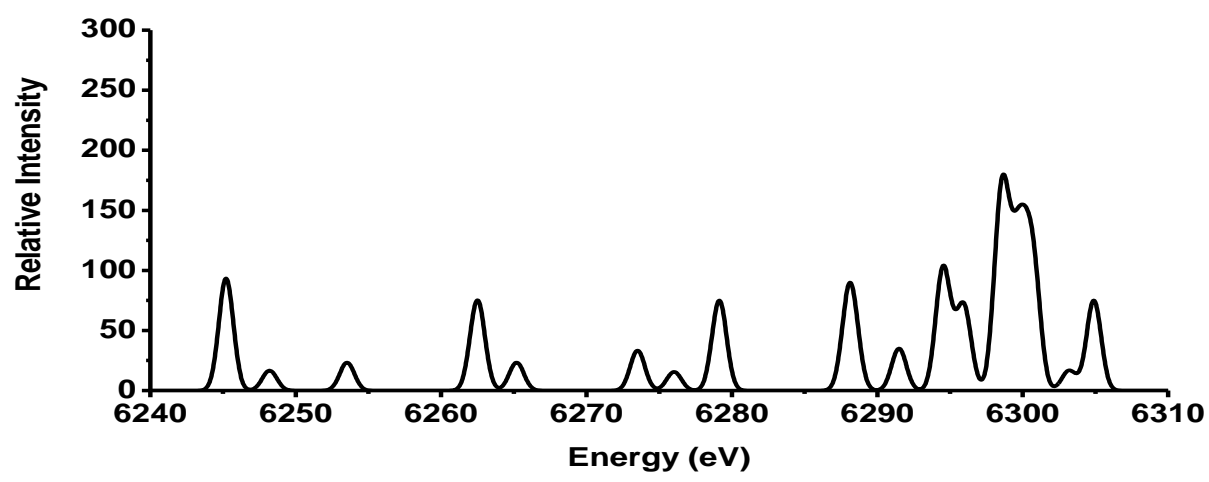

Fig.3 (b) The L-emission satellite spectra obtained as the envelope of 30 Gaussian curves for the 30 electronic transitions given in table 1 . The Gaussian curve for each of the transitions has $\sigma=0.5$ and $\Delta \mathrm{E}=0.03 \mathrm{eV}$. 


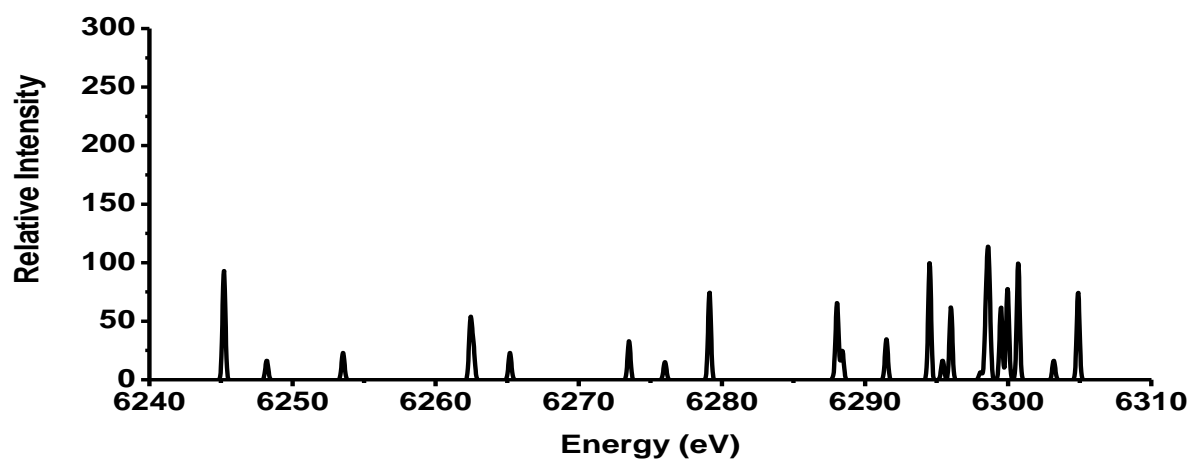

Fig.3(c) The L-emission satellite spectra obtained as the envelope of 30 Gaussian curves for the 30 electronic transitions given in table 1 . The Gaussian curve for each of the transitions has $\sigma=0.1$ and $\Delta \mathrm{E}=0.03 \mathrm{eV}$.

\section{COMPUTER PROGRAM FOR COMPUTING THEORETICAL X-RAY SATELLITE SPECTRA}

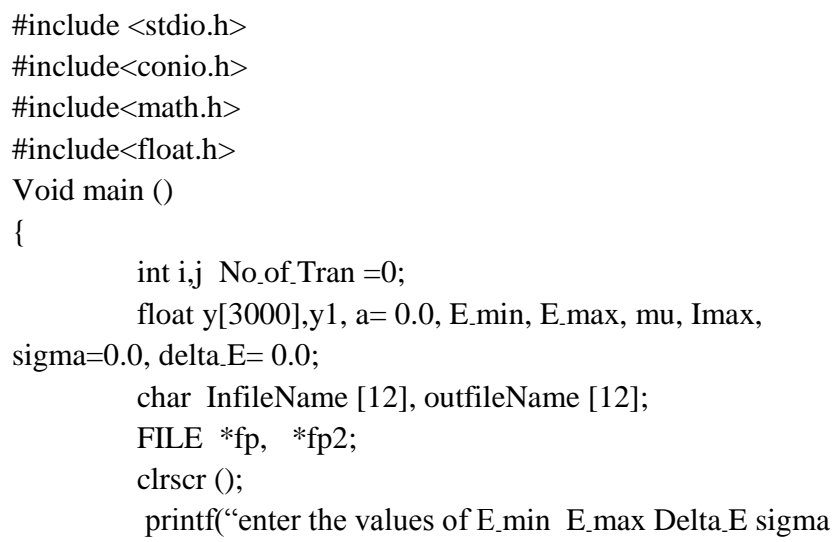

else $\}$

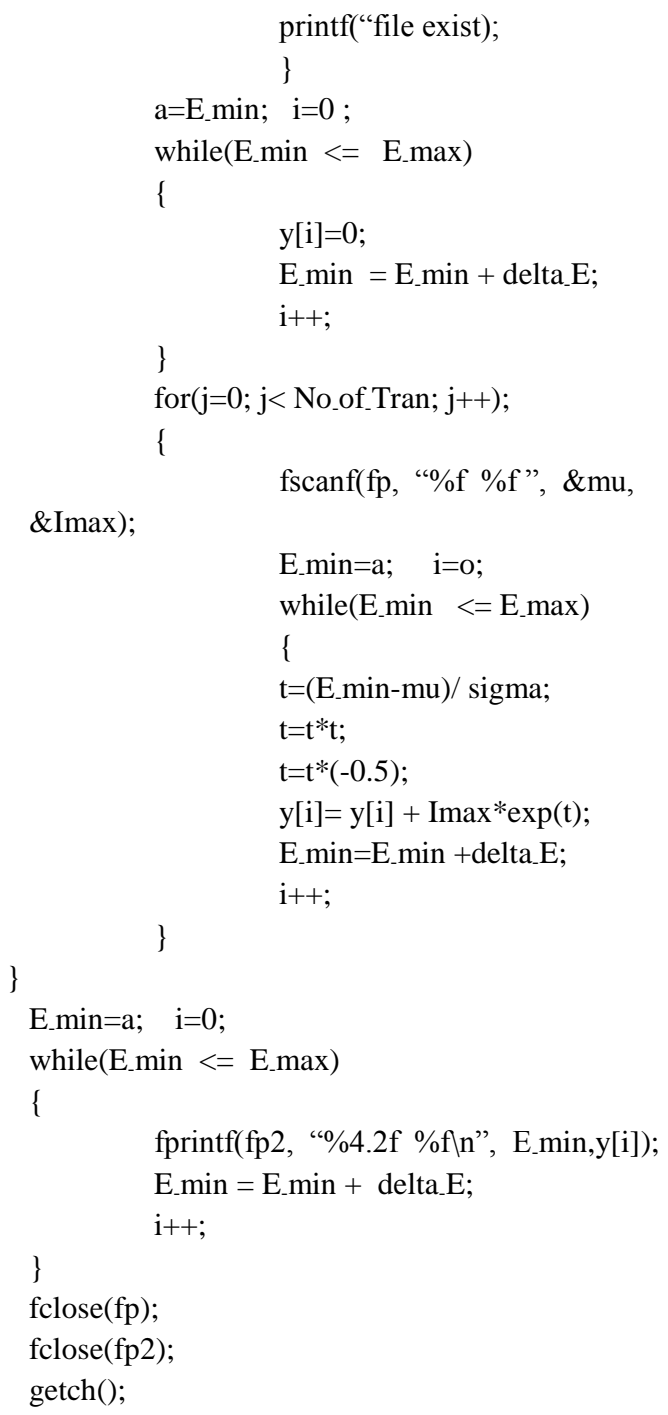




\section{References}

[1] B D Shrivastava and Uma Shrivastava, $2 p_{3 / 2}^{-1} 3 x^{-1}-3 x^{-1} 3 d_{3 / 2}^{-1} x$-ray satellites in the L $\alpha_{2}$ region, X-ray Spectrom., Vol. 34, pp179-182, 2005.

[2] Uma Shrivastava, Some investigations in X-ray satellite spectra, $\mathrm{Ph}$. D. Thesis (unpublished), Vikram University, Ujjain, pp.5758, 2002.

[3] Rajeev Trivedi, Some theoretical investigations in X-ray satellite spectra, Ph.D. Thesis, Vikram University, Ujjain, pp 68-69, 2008.

[4] Renuka Kendurkar, Some investigations in X-ray satellite spectra, Ph.D. Thesis (unpublished), Vikram University, Ujjain,pp.22 \& 66, 2010.

[5] M R Spiegel, , Theory and Problems of Statistics, Schaum's Outline Series, pp122, 1981.

[6] F Parente, M H Chen, B Crasemann and H Mark, $L$ X-ray satellite energies, At. Data Nucl. Data Tables, 26, pp 383, 1981.

\section{AUTHORS PROFILE}

Dr. B. D. Shrivastava obtained his M.Sc. from University of Rajasthan, Jaipur in 1960. He joined School of Physics, Vikram University, Ujjain as Lecturer in 1961. He was awarded Ph.D. from Vikram University, Ujjain in 1973. He worked as Professor of Physics in the School of Studies in Physics, Vikram University, Ujjain and retired in 2002. Even after his retirement he is actively engaged in research work. His fields of interest are: $\mathrm{X}$-ray absorption spectroscopy and X-ray emission spectroscopy. He has published more than 142 papers in various National and International Journals. He was awarded Fulbright fellowship in 1987-88. He worked with Prof. Edward Stern in the University of Washington, Seattle, USA. $\mathrm{He}$ also worked at Universidad Autónoma de Madrid, Madrid, Spain, under the scheme of IndoSpanish fellowship program. 26 students have obtained Ph.D. degree under his supervision. He is one of the editors of the book "X-ray spectroscopy and allied areas" published by Narosa Publishing House, New Delhi, 1988 with ISBN No. 81-7319243-X. He was also nominated as member International Advisory Committee, $5^{\text {th }}$ International Conference on X-ray Absorption Fine Structure, University of Washington, Seattle, U.S.A. from Aug.21-26,1988. He was nominated as member of the committee for preparing panel for Vice-Chancellor, Devi Ahilya University, Indore, the meeting of which was held at New Delhi on 19-062010.

Dr. Ramji Yadav obtained B.Tech. in Computer Engineering in 1992 from College of Technology, Gobind Ballabh Pant University, Pantnagar, Nainital, U.P. He did M.Tech. in Computer Science in 1995 from School of Computer Science, DAVV, Indore. M.P. He did Ph.D. in 2011 from School of Computer Science, Faculty of Engineering and Technology, Jiwaji University Gwalior. He has experience of 23 years of teaching computer science to PG and UG classes. He has been resource person in refresher courses and workshops for teachers. Presently, he is looking after the Institute of Computer Science, Vikram University Ujjain, M.P. as Director. He has published 9 research papers in national and international journals and conferences. He has been chairman and member of BOS in Vikram University as well as in other universities.

Ms. Renuka Kendurkar obtained Bachelor of Science from Madhav Science College, Ujjain in the year 1992 and Master of Science in Physics from School of Studies in Physics, Vikram University, Ujjain (M.P.) India, in 1994. She obtained Ph.D. in Physics in 2011. Her main research work focuses on X-ray emission spectroscopy. She has 20 years of teaching experience and 10 years of research experience. She has published 7 research papers.

Mrs. Falguni Shrivastava obtained Bachelor of Science and Master of Science from Holkar Science College, Indore (M.P.) India, in year 2007 and 2010 respectively. She is currently pursuing Ph.D. since 2013. Her main research work focuses on $\mathrm{X}$-ray emission spectroscopy. She has 2 years of teaching experience and 4 years of research experience. 